\title{
Grand challenges in extreme microbiology
}

\section{Andreas Teske*}

UNC Chapel Hill, Chapel Hill, NC, USA

*Correspondence: teske@email.unc.edu
As the microbiological exploration of our home planet intensifies, we discover that an ever-growing range of extreme environments harbors novel types of microorganisms. Wherever we look, previously unknown bacteria, archaea, and protists appear into view. Scientific perceptions of the microbial world and its "extreme" fringes have changed with increasing pace and with unforeseen consequences - and lead directly toward the grand research challenges for the future.

\section{DIVERSITY}

Not long ago, microbial ecosystems in extreme environments were regarded as textbook examples of simplicity; a second and a third look with improved microbiological tools revealed them as model cases of complexity, with highly differentiated physiologies and divergent evolutionary lineages on the highest taxonomic levels. For example, hypersaline or thermophilic cyanobacterial mats were considered relatively easily structured microbial ecosystems, dominated by several types of microscopically dominant, generally cultivable cyanobacteria or Chloroflexi; the physiology of these representative species and isolates explained the functioning of the mat ecosystem (Cohen and Rosenberg, 1989). With increasingly integrated molecular and physiological studies, the few morphotypes and representative cultured species of "simply" structured microbial mat ecosystems gave way to increasingly complex, ecophysiologically and molecularly differentiated population mosaics and activity gradients of microorganisms that make optimal use of every niche, every gradation of chemistry or temperature in their microenvironment (Ward et al., 1998). Recently, attempts to fully describe the microbial diversity of hypersaline cyanobacterial mats on the level of $16 \mathrm{~S}$ rRNA genes yielded discoveries of deep, ancient evolutionary lineages in all domains of life (Robertson et al., 2009). Even the most comprehensive sequencing surveys may not account for all genetic variation and phylogenetic depth of natural microbial communities; do we now, with next-generation sequencing techniques in hand, finally map the outer ends of microbial biodiversity? The idea of "simple" microbial ecosystems in extreme environments is gone; microbiologists are faced with the grand challenge of accounting for the full extent and diversity of microbial life on Earth.

\section{LIMITS OF LIFE}

The last years have seen an unprecedented flowering of extreme microbiology in all its aspects, including molecular studies, cultivations of novel microorganisms, and surveys of new habitats; in consequence, the field has moved from a niche specialty toward the mainstream of microbiology. The abundant discoveries of new habitats and new organisms have progressed beyond the accumulation of individual new findings and results, toward the search for a general framework of what constitutes "extreme" microorganisms. A common characteristic of microorganisms that live under extreme physical and chemical conditions might be energy stress, in the sense that almost all metabolically available energy is consumed by maintenance and upkeep of essential cell structures, biomolecules, enzymes, and pathways; this problem is compounded by the poor energy yields of many microbial metabolisms under extreme chemical regimes of electron acceptor or donor limitation (Valentine, 2007). The unifying theme of energy limitation is taken up and developed further by the fields of biogeochemistry and Astrobiology (Hoehler et al., 2007). Here, microbial life is viewed as pervasive on this and other planets, as long as suitable and accessible energy sources for microbial metabolism exist. Seen in this light, the biology of extreme microorganisms has come very far from its beginnings as an intriguing collection of strange organisms; extreme microbiology becomes an exploratory endeavor and research challenge of mapping the outer boundaries of life itself.

\section{CULTIVATIONS}

The full diversity of the microbial world needs to be explored by cultivating new microorganisms, and by studying them as enrichments or in pure culture. Exploring the physiology of a previously uncultured microorganism - its energy metabolism, preferred carbon and nutrient sources, responses to environmental stimuli and stressors, and strategies for growth and survival - cannot be replaced by any other approach. A thorough knowledge of the organism in culture is essential to groundtruth sequence analyses and genomic or transscriptomic studies. A classical example is the isolation of Geothermobacterium ferrireducens, an iron-reducing, hyperthermophilic bacterium that is most closely related to the hyperthermophilic sulfatereducing genus Thermodesulfobacterium. A molecular identification, without cultivation, would have mistaken this bacterium as a sulfate reducer (Kashefi et al., 2002). Beyond ground-truthing the "omics" or incrementally extending the diversity of microbial life in culture, isolations or stable enrichments can lead to entirely surprising, original discoveries that are impossible to anticipate or to plan. As a famous example, consider the sole known stable coculture of two hyperthermophilic archaea, Ignicoccus hospitalis and its "symbiont," Nanoarchaeum equitans; in the former archaeon, information processing and protein synthesis are spatially separated from energy conservation, a unique case in the microbial world (Küper et al., 2010), Obviously, catching up with Mother Nature's expertise in cultivation is a highly rewarding challenge for the future of Extreme Microbiology.

These three grand research challenges for the future are certainly not the only ones, but they demonstrate that the unifying perspectives and big-picture questions of extreme microbiology reach beyond disciplinary boundaries. To conclude with an understatement, the outlook of extreme microbiology and its future contributions to the life sciences is highly promising. 


\section{REFERENCES}

Cohen, Y., and Rosenberg, E. (1989). Microbial Mats: Physiological Ecologies of Benthic Microbial Communities. Washington, DC: ASM Press.

Hoehler, T. M, Amend, J. P., and Shock, E. L. (2007). Introduction - a "Follow the energy" approach for astrobiology. Astrobiology 7, 819-823.

Kashefi, K., Holmes, D. E., Reysenbach, A.-L., and Lovley, D. R. (2002).Use of Fe(III) as an electron acceptor to recover previously uncultured hyperthermophiles: isolation and characterization of Geothermobacterium ferrireducens gen. nov., sp. nov. Appl. Environ. Microbiol. 68:1735-1742.

Küper, U., Meyer, C., Müller, V., Rachel, R., and Huber, H. (2010). Energized outer membrane and spatial separation of metabolic processes in the hyperthermophilic archaeon Ignicoccus hospitalis. Proc. Natl. Acad. Sci. U.S.A. 107, 3152-3156.

Robertson, C. E., Spear, J. R., Harris, J. K., and Pace, N. R. (2009). Diversity and stratification of archaea in a hypersaline microbial mat. Appl. Environ. Microbiol. 75, 1801-1810.

Valentine, D. A. (2007). Adaptations to energy stress dictate the ecology and evolution of the archaea. Nat. Rev. Microbiol. 5, 316-323.

Ward, D. M., Ferris, M. J., Nold, S. C., and Bateson, M. M. (1998). A natural view of microbial biodiversity within hot spring cyanobacterial mat communities. Microbiol. Mol. Biol. Rev. 62, 1353-1370.
Received:01 September 2010; accepted:07 September 2010; published online: 04 October 2010

Citation: Teske A (2010) Grand challenges in extreme microbiology. Front. Microbio. 1:111. doi: 10.3389/ fmicb.2010.00111

This article was submitted to Frontiers in Extreme Microbiology, a specialty of Frontiers in Microbiology.

Copyright (C) 2010 Teske. This is an open-access article subject to an exclusive license agreement between the authors and the Frontiers Research Foundation, which permits unrestricted use, distribution, and reproduction in any medium, provided the original authors and source are credited. 